\title{
Comparison of an Australian food-frequency questionnaire with diet records: implications for nutrition surveillance
}

\author{
GL Ambrosini ${ }^{1, *}$, D Mackerras ${ }^{2}, \mathrm{NH}$ de Klerk ${ }^{1}$ and AW Musk ${ }^{1,3}$ \\ ${ }^{1}$ School of Population Health, University of Western Australia, Crawley, Western Australia 6009, Australia: \\ ${ }^{2}$ Menzies School of Health Research, Casuarina, Northern Territory 081 1, Australia: ${ }^{3}$ Department of Respiratory \\ Medicine, Sir Charles Gairdner Hospital, Nedlands, Western Australia 6009, Australia
}

Submitted 9 April 2002: Accepted 21 October 2002

\begin{abstract}
Objectives: To compare a widely used Australian food-frequency questionnaire (FFQ) with diet records and consider the results in relation to its use in nutrition surveillance. Design: Inter-method reliability study.

Setting: A randomised trial in subjects with past asbestos exposure.

Subjects: Seventy-two adults living in Western Australia.

Methods: A semi-quantitative FFQ developed by the Commonwealth Scientific Industrial Research Organisation in South Australia was administered after the completion of four 7-day diet records (DRs).

Results: Mean agreement between methods was not significantly different from 100\% for many nutrients, but the limits of agreement indicated that, at the individual level, the FFQ over- or underestimated the DR by at least 50\%. Mean agreement between methods decreased significantly with increasing intakes for the majority of nutrients. Pearson's correlation coefficients were less informative indicators of agreement compared with the limits of agreement.

Conclusions: These results indicate poor agreement between the FFQ and DR when estimating absolute intakes. Therefore, comparing intakes collected using this FFQ with specific cut-off points such as Recommended Dietary Intakes for nutrition surveillance may lead to seriously flawed conclusions about population intakes.
\end{abstract}

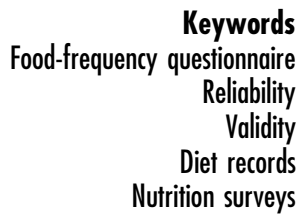

Food-frequency questionnaires (FFQs) were designed for epidemiological studies to estimate usual intake for ranking individuals when calculating diet-disease risks. However, an FFQ capable of ranking intake does not necessarily provide good absolute measures of intake ${ }^{1}$. Good estimates of absolute levels of intake, rather than correct ranks, are required for formulating public health recommendations ${ }^{2}$. For population monitoring, a tool that can detect changes in population intake may often be sufficient. However, more quantitative results, such as the proportion with low intakes of certain nutrients, may also be desired.

Most studies examining the properties of FFQs have assessed their ability to rank subjects with the intent to adjust observed risk ratios for error. Few studies have examined their ability to measure absolute nutrient or food intakes. Despite this, FFQs are becoming quite widely used in national surveys and other surveillance methods. In this paper, we compare the absolute nutrient intakes from a widely used Australian FFQ with multiple 7-day diet records (DRs), and consider some of the implications of using FFQs for quantitative nutrition surveillance.

\section{Background}

In the early 1980s, the Commonwealth Scientific Industrial Research Organisation (CSIRO) in South Australia developed a semi-quantitative FFQ ${ }^{3,4}$. This FFQ collects openended frequency and portion size information on nearly 200 items, including single foods, mixed dishes, beverages and alcohol ${ }^{3,4}$. Most of the food items remain constant but a small number are changed to match the focus of the study it is used in. An example of a medium portion size for each item is given in household units based on weighed diet records collected in previous work ${ }^{5}$ and subjects record whether their own servings are small, medium or large in relation to the nominated amount. Other information including type of fat used and the use of added salt is also collected.

Slightly modified versions of this questionnaire have been used to examine the influence of diet on the risk of stroke and coronary heart disease ${ }^{6}$, asthma in children ${ }^{7}$, pancreatic cancer $^{8}$, colorectal cancer ${ }^{9-11}$ and breast cancer $^{12}$. Modified versions have also been used to assess the achievement of Recommended Dietary Intakes (RDIs) in children ${ }^{13}$, adults ${ }^{13-15}$ and the elderly ${ }^{16-18}$, the 
contribution of nutrients and energy in the diets of children $^{19}$, adults ${ }^{20,21}$ and diabetics ${ }^{22,23}$, and to monitor dietary changes as indicators of the effectiveness of health promotion programmes ${ }^{24,25}$. We examined the 'Questan' version with 172 items, which was administered in a statewide survey of the state of Victoria in $1985^{21}$ and has been used in a national postal survey every 5 years since 1988 to assess usual food and nutrient intakes ${ }^{26}$.

The repeatability of this FFQ has been reported ${ }^{13,27}$ but little information about its validity relative to other dietary methods has been published, despite its extensive use. The limited reports that exist have examined only a few nutrients ${ }^{3,23,28,29}$, were conducted with female student dietitians ${ }^{29}$ or compared total fruit and vegetable intakes only ${ }^{30}$. In this paper we compare all nutrient intakes from the 'Questan' version with multiple 7-day DRs, in a more general sample of the adult population. Although this type of study is commonly referred to as a validation study, it is more correctly described as an inter-method reliability study $^{31}$. Our primary analysis uses the limits of agreement method $^{32}$, but we also report the widely used correlation coefficient for comparison with previous work.

\section{Methods}

\section{Subjects}

This study was conducted within a randomised trial examining the efficacy of $\beta$-carotene and retinol supplements in reducing the risk of malignant mesothelioma and other cancers. Trial participants were 3240 people aged 8 to 87 years ( $80 \%$ men) recruited from two large cohorts of ex-workers and ex-residents of a blue asbestos mining town in Western Australia ${ }^{33}$. All of the 570 people enrolling during the sixth month of the trial were asked if they would provide four 7-day DRs over the following year, except where language or literacy difficulties were apparent at the time of interview. All subjects gave informed consent and the study was approved by the Human Research Ethics Committee of the University of Western Australia and the Clinical Drug Trials Committee of the Sir Charles Gairdner Hospital, Nedlands, Western Australia.

\section{Dietary methods}

Subjects agreeing to participate in the reliability study were shown how to record everything consumed for seven consecutive days using household units (not weights) and were provided with measuring instruments and record sheets at their enrolment interview. One 7-day DR was completed in early December 1990 and the others in April, July and October of 1991. Participants were followed up by telephone two weeks after each commencement date to check their progress and to set a date for person-to-person checking of their DR.

After the completion of their fourth 7-day DR, the FFQ was posted to subjects to complete at home. Completed questionnaires were returned by post and any missing information in the FFQ was followed up with the subject by telephone.

All DRs were entered into a database by the same nutritionist (G.L.A.) using Xyris Diet/1 software ${ }^{34}$ and daily nutrient intakes were calculated using Australian Food Composition Tables (NUTTAB91-92) ${ }^{35}$. Mean daily nutrient intakes from the four DRs combined were used for agreement analyses. Mean daily nutrient intakes from the FFQ were calculated by CSIRO Health Sciences and Nutrition (South Australia), utilising their automated system for linking the questionnaire with the same edition of the Australian Food Composition Tables ${ }^{4}$. The percentages of energy derived from fat and carbohydrate were calculated, as these are common surveillance indicators. Nutrient intakes from supplements were excluded. Sodium was excluded because discretionary salt use was not ascertained in the DR. All nutrient intake data were transformed before analyses using natural logarithms to overcome skewed distributions.

\section{Internal validity}

The ratio of energy intake to basal metabolic rate (EI/BMR) was used to assess underreporting of total intakes. BMR was calculated using Food and Agriculture Organization/ World Health Organization/United Nations University equations adjusting for sex, age and weight ${ }^{36}$. Estimates of energy intake from each dietary method were checked for extremes. Energy intakes less than $3360 \mathrm{~kJ}$ or above $21000 \mathrm{~kJ}$ were considered implausible $\mathrm{e}^{37}$.

The characteristics of reliability study participants were compared with those of other trial participants who were invited but declined to participate in the reliability study, using Fisher's exact tests to compare proportions and $t$-tests to compare continuous variables as appropriate.

\section{Agreement}

The correlation coefficient describes association but not agreement; therefore the limits of agreement (LOA) were calculated to compare the two dietary methods ${ }^{38,39}$. For each subject, the difference between the FFQ and DR (FFQ-DR) and the average of the FFQ and DR $((\mathrm{FFQ}+\mathrm{DR}) / 2)$ was calculated. Plotting these two results allows visual assessment as to whether agreement between methods varies across the range of intakes and, if so, in which direction. This was formally tested by fitting the regression line of differences $\left(H_{\mathrm{O}}: \beta=0\right.$, $\alpha=0.05)$. The LOA define the boundaries within which $95 \%$ of all the differences between methods are expected to fall and were calculated as: mean agreement (or mean of all differences) $\pm t_{(n-1,0.025)}$ standard deviation of the differences $^{38}$. Acceptable LOA are arbitrary, but should depend upon the required sensitivity of a measure or $\operatorname{method}^{38}$.

Because dietary intakes were log-transformed for the agreement analysis, anti-logging rendered mean 
agreement, the LOA and their 95\% confidence limits as a ratio, i.e. a multiple of the FFQ relative to the DR. All were expressed as a percentage, with 100\% indicating exact agreement $^{38}$. For example, mean agreement of $120 \%$ indicated that, on average, FFQ estimates were 1.2 times greater than the DR estimate. LOA of 50-200\% would indicate that $95 \%$ of all subjects' FFQ estimates were between one-half and two times their DR estimate. We summarised agreement between methods according to the LOA and any dependency between mean agreement and the magnitude of intake (i.e. regression slope of differences $\neq 0$ ). This was done separately for men and women.

Given the individual variation in usual diet, it is unlikely that agreement between two different dietary methods will be 100\%. However, even when mean agreement is close to $100 \%$, very wide LOA indicate that individual differences between dietary methods may be unacceptably large. A dependency between agreement and magnitude of intake indicates that the error in FFQ estimates varies across the range of intakes. This can lead to differential misclassification of intake, which introduces error around any cut-off points applied to absolute intakes, such as those used to make comparisons with RDIs. It also reduces the ranking ability of the FFQ, and the precision of diet-disease risk estimates. Furthermore, when FFQ errors vary across intakes it is inappropriate to apply a single calibration factor across the range of intakes.

To compare our results with other studies on the same FFQ, we also calculated Pearson's correlation coefficients between the natural log-transformed intakes from each method using PROC CORR in SAS ${ }^{40}$.

\section{Results}

Of 118 volunteers, 83 people ( 57 men and 26 women) successfully completed four 7-day DRs and 72 of these ( 48 men and 24 women) completed the FFQ after their last 7-day DR. Over half of those withdrawing from the reliability study did so before completing their first 7-day DR. The most common reasons for not completing all four diet records or subsequent questionnaires were the commitment required $(n=31)$ and withdrawal from the trial due to side-effects or non-compliance $(n=11)$. The 11 subjects (nine men and two women) not completing the FFQ after their DRs did so mostly due to reluctance to complete more questionnaires. However, they were more likely to be users of dietary supplements before entering the trial and never to have smoked, compared with other reliability subjects and trial subjects (comparisons not shown). There were no significant differences in mean age or body mass index (BMI), the proportions of men and women, current smokers, subjects with BMI $>25 \mathrm{~kg} \mathrm{~m}^{-2}$ or supplement users, between reliability study participants and those who declined to participate (Table 1).

Typical EI/BMR in a Western population is 1.55 for men and 1.56 for women ${ }^{42}$. For those completing the DRs and the FFQ, EI/BMR was 1.3 for both men and women using FFQ intakes, and 1.4 and 1.2, respectively, using DR intakes. One subject had to be excluded from the reliability analysis, because his FFQ energy estimate was above $25000 \mathrm{~kJ}$ and could not be explained by his activity level or BMI.

For men the FFQ estimate ranged from $70 \%$ ( $\beta$-carotene) to $127 \%$ (sugars) of the DR estimate (Table 2). As indicated by the confidence intervals for mean agreement, many of these discrepancies were statistically significant. The LOA indicate that the FFQ could either underestimate or overestimate many nutrient intakes by at least 50\% of the DR estimate. For example, a man with a DR energy intake of $5000 \mathrm{~kJ}$ could have an FFQ energy estimate anywhere between $2850 \mathrm{~kJ}$ (57\%) and $7000 \mathrm{~kJ}$ (140\%). Particularly wide LOA were observed for alcohol, $\beta$-carotene, polyunsaturated fat, retinol, retinol equivalents, sugars and vitamin C.

For women (Table 3), mean agreement ranged from $90 \%$ (saturated fat) to $163 \%$ (vitamin C). Although the $95 \%$

Table 1 Baseline characteristics of subjects in the reliability study and those who declined to participate

\begin{tabular}{|c|c|c|c|c|}
\hline & \multicolumn{2}{|c|}{ Men } & \multicolumn{2}{|c|}{ Women } \\
\hline & Declined* & Participants & Declined $^{*}$ & Participants \\
\hline$n$ & 389 & 48 & 109 & 24 \\
\hline$\%$ & 78 & 67 & 22 & 33 \\
\hline Age (years), mean \pm SD & $54 \pm 11$ & $55 \pm 10$ & $50 \pm 13$ & $49 \pm 11$ \\
\hline $\mathrm{BMI}\left(\mathrm{kg} \mathrm{m}^{-2}\right)$, mean $\pm \mathrm{SD}$ & $28 \pm 4$ & $27 \pm 3$ & $27 \pm 4$ & $28 \pm 5$ \\
\hline $\mathrm{BMI}>25 \mathrm{~kg} \mathrm{~m}^{-2}(\%)$ & 77 & 78 & 63 & 75 \\
\hline Never smoked (\%) & 23 & 30 & 50 & 68 \\
\hline Ex-smoker (\%) & 51 & 50 & 30 & 16 \\
\hline Current smoker (\%) & 26 & 20 & 20 & 16 \\
\hline \multicolumn{5}{|l|}{ Cigarettes smoked per day by } \\
\hline Taking a supplement prior to study† (\%) & 9 & 4 & 14 & 8 \\
\hline
\end{tabular}

SD - standard deviation; BMI - body mass index.

*Participants who were invited but chose not to participate in the reliability study, including those who agreed and later dropped out.

† Supplement use was assessed using a different FFQ developed for the trial ${ }^{41}$. 


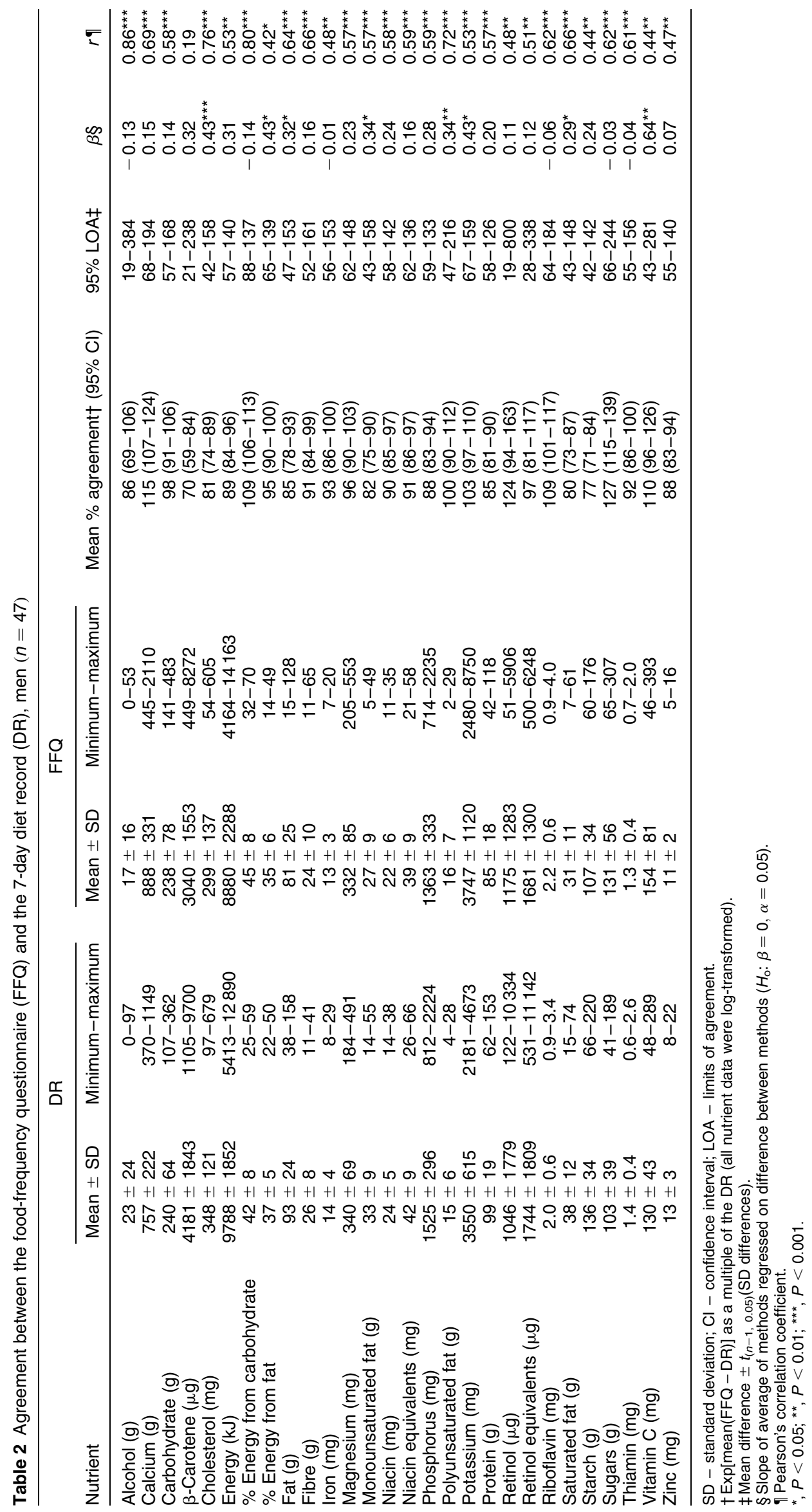


confidence intervals and LOA were wider for the majority of nutrients reported by women in comparison to men, this may be a result of the smaller number of women rather than real sex differences.

Many nutrients showed a significant dependency between agreement and the magnitude of intake (Fig. 1 shows an example). Men reported fewer nutrients showing this dependency and had smaller regression coefficients (slopes) than women. Most often the dependency was positive, indicating that agreement became worse with increasing intakes. A negative slope, as seen for alcohol, meant that agreement was worse at lower levels of intake; however, none of these was significant. Expressing carbohydrate and fat as percentages of energy intake improved mean agreement and the LOA for fat only.

Criteria for assessing the adequacy of agreement are arbitrary. We selected (1) constant agreement across levels of intake, i.e. no significant slope in the regression line of differences, and (2) LOA not exceeding 50-200\%, i.e. the FFQ yielded up to one-half or double the DR value. According to these criteria, agreement was adequate for all nutrients reported by men except for alcohol, $\beta$-carotene, cholesterol, total fat and all fat types, percentage of energy from fat, potassium, retinol, retinol equivalents, sugars and vitamin C. In women, only starch and percentage of energy from carbohydrate showed acceptable agreement. In contrast, most of the correlation coefficients between the two methods (for both men and women) were strong enough that they would often be interpreted as indicating 'good agreement'. Generally, the nutrients with the strongest correlations were not the ones identified as having adequate agreement, e.g. alcohol, cholesterol, polyunsaturated fat. Correlations were generally stronger in the men.

\section{Discussion}

These analyses show that, in a sample of free-living adults, broad statements cannot be made about whether the FFQ over- or underestimates intakes compared with the DR. Although mean agreement was close to $100 \%$ for most nutrients, this indicates that the two methods agree at the mean; it does not indicate $100 \%$ agreement across the scale of intakes. More importantly though, for many nutrients, levels of agreement were not constant but decreased significantly with increasing intakes. Like many FFQs, agreement between vitamin A intakes was poorest, and our results indicate large potential discrepancies between intakes of alcohol, $\beta$-carotene, cholesterol, all fat types and percentage of energy from fat, potassium, sugars and vitamin C, for both men and women. There were important differences in mean agreement between the sexes that are not necessarily related to the sample size. However, the wider LOA observed among women are probably due to their smaller numbers creating wider confidence limits.

This was the third FFQ completed by subjects in one year (but the first CSIRO FFQ), as participants completed another brief FFQ at entry to the trial and at the end of their DRs, for another reliability study ${ }^{41}$. Any training effect would have had either no effect on agreement (where subjects may have more efficiently recalled the same errors) or improved subjects' recall. Hence, the agreement observed in this study might be better than would be expected if the FFQ were used in the wider population, among participants who had not completed an FFQ or diet record before.

None of the previous studies examining the CSIRO FFQ have used the LOA method. Pearson's correlation coefficients reported in these earlier studies ranged from 0.38 for protein ${ }^{28}$ to 0.60 for saturated fat and calcium ${ }^{23,28}$. Spearman's rank correlations ranged from 0.34 for protein to 0.83 for total $\mathrm{fat}^{29}$. The one study that compared all FFQ nutrients with 12 days of weighed DRs for men and women separately reported kappa statistics less than 0.4 for most nutrients, indicating that classification agreement was generally poor between the two methods ${ }^{43}$. Median differences and interquartile ranges in that study were greatest for carbohydrates, sugars, fibre, calcium, vitamin $\mathrm{C}, \beta$-carotene and vitamin $\mathrm{A}$, and agreement was reported as slightly better among men.

The correlation coefficients from these previously published studies are similar to our results in men except that our correlation coefficients for protein, carbohydrates, alcohol and calcium were generally higher. Although the magnitude of a correlation coefficient depends on the range studied, these results may indicate that the patterns observed in our study are not unusual. Therefore our LOA results might reflect the existence of poor agreement in other Australian sub-populations that have used this FFQ. Our results also indicate important differences in FFQ performance between men and women. As our numbers were small, this should be confirmed before making a definite conclusion.

Nutrient intakes from another Australian FFQ, that developed by the Anti-Cancer Council of Victoria (ACCV), have been compared with those from a 7-day weighed DR using the LOA method ${ }^{44}$. In marked contrast to our findings, there were no variations in the level of agreement across levels of intake for any of the 27 nutrients assessed. A notable difference between the ACCV and CSIRO questionnaires is the use of questions in the ACCV FFQ to calibrate fruit and vegetable intakes according to the total number of portions consumed per day. This difference may be important, because another study found that the long list of fruits and vegetables in the CSIRO FFQ yields much higher intake frequencies than the shorter list ${ }^{30}$. Mean agreement and LOA were not presented as percentages in the ACCV study, but the authors noted as did we - that LOA outside 50-100\% were observed. 


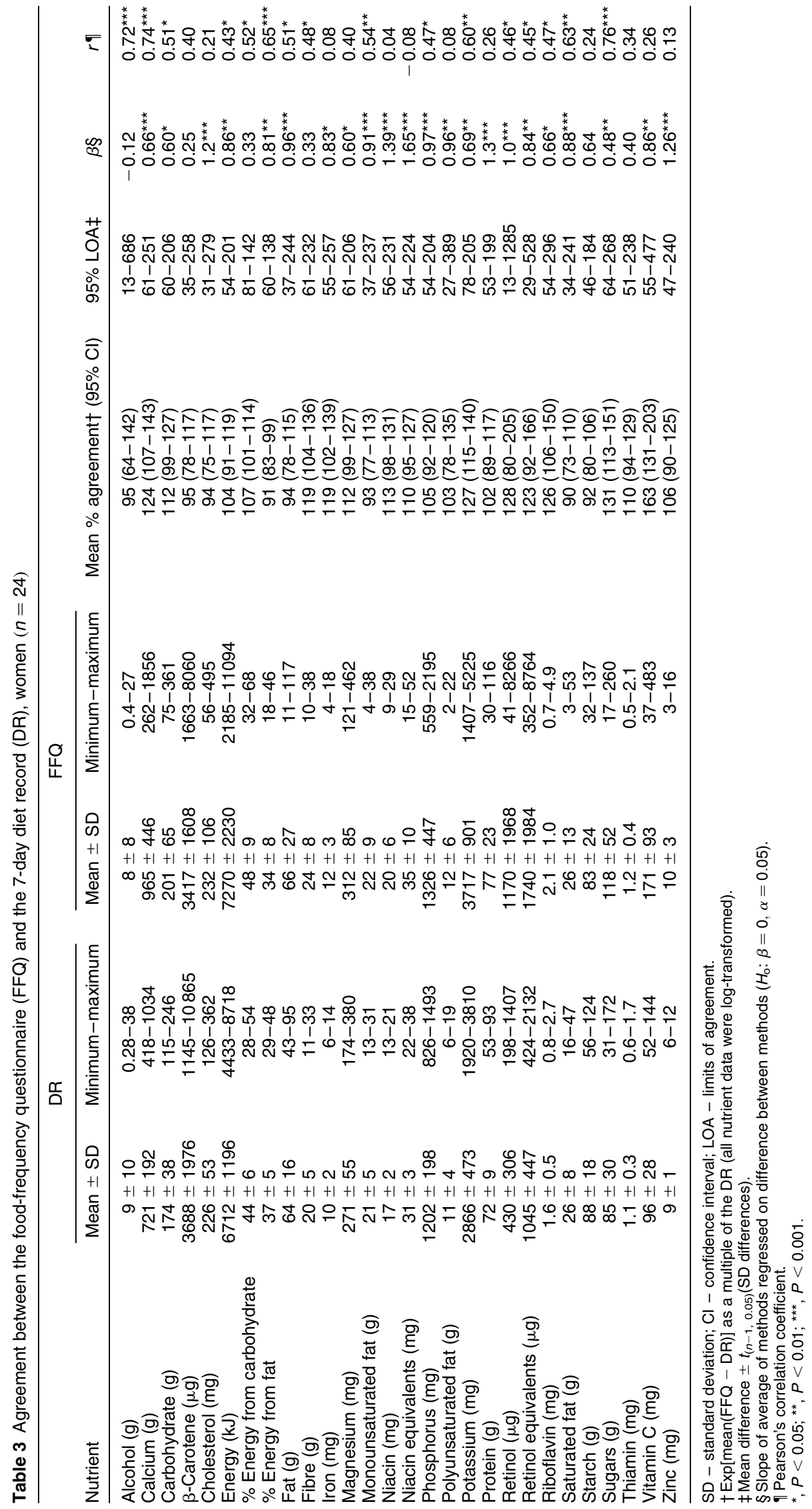




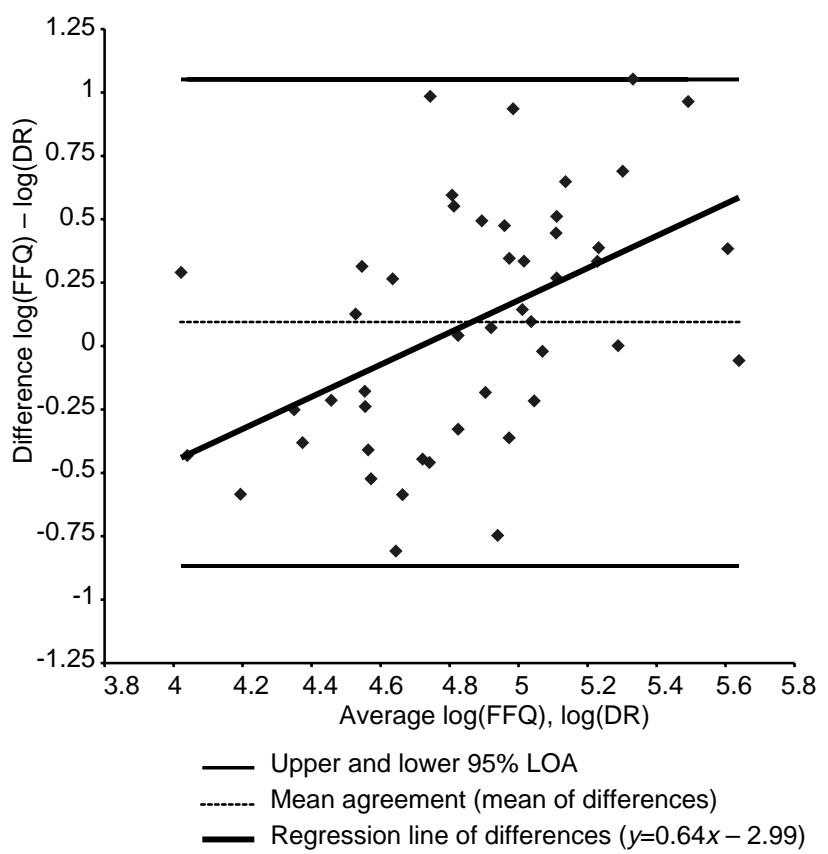

Fig. 1 Limits of agreement (LOA) for vitamin $C$ intake, men $(n=47)$. FFQ - food-frequency questionnaire; DR - 7-day diet record

The ACCV findings were also similar to ours in that agreement was poor for vitamin $\mathrm{A}, \beta$-carotene, vitamin $\mathrm{C}$, all types of fat, cholesterol and calcium. Although the ACCV study included women only, it confirms that wide LOA observed between an FFQ and DR are not unusual.

It can be argued that dietary intakes used to assess the achievement of RDIs should be obtained in the same way that RDIs have been established, i.e. using duplicate weighed diet records. The large discrepancies observed between the FFQ and DR in this study indicate that FFQ intakes do not permit confident assessments of absolute intake against specific cut-off points such as RDIs or dietary goals. However, this FFQ is used regularly for such monitoring. One important drawback for monitoring purposes is that intakes are generally underestimated by both methods (indicated by the low EI/BMR) and this will lead to general overestimation of the proportion of the population with low intakes. As the underestimation was similar in both methods, it is reasonable to compare the extent of the disparity. For example, the proportion of women with intakes below the RDI for calcium was 58\% and $92 \%$ for the FFQ and DR, respectively. Similarly, the proportion consuming more than 30\% of energy as fat according to FFQ estimates was $90 \%$ for men and $71 \%$ for women, and according to the DR it was $92 \%$ for men and $96 \%$ for women. These problems also indicate that quantitative targets for population intakes to prevent chronic disease cannot be derived from epidemiological studies that have used FFQs unless the errors have been corrected.

FFQs represent a useful, economical and rapid method for large nutrition surveys compared with more labour-intensive methods like the diet record. However, FFQs require further evaluation if they are to be used quantitatively, and their estimates can be calibrated using well-conducted reliability studies and simple regression techniques $^{32}$. The limits of agreement method used here shows significant discrepancies between FFQ intakes and a diet record, and that agreement between methods can vary significantly across levels of intake in the population being studied. Although this study is small, it also raises the possibility that this FFQ performs differently in men and women, and that therefore intakes from this, and perhaps other FFQs, are not automatically comparable between the sexes. The degree of agreement, or lack of it, between the FFQ and DR examined here highlights the potential problems with using food-frequency questionnaires to assess absolute dietary intakes and other specific properties of dietary intake for quantitative surveillance purposes.

\section{Acknowledgements}

The authors wish to thank Caroline Hickling, Irene Foundas, Fiona Smith, Lynne Watts, Nola Olsen and Jan Sleith for their assistance with the data collection for this study.

\section{References}

1 Sempos C. Some limitations of semi-quantitative food frequency questionnaires [invited commentary]. Am. J. Epidemiol. 1992; 135: 1127-32.

2 Lissner L, Heitman BL, Lindroos AK. Measuring intake in free-living human subjects: a question of bias. Proc. Nutr. Soc. 1998; 57: 333-9.

3 Baghurst KI, Baghurst PA. The measurement of usual dietary intake in individuals and groups. Trans. Menzies Found. 1981; 3: 139-60.

4 Baghurst KI, Record SJ. A computerised dietary analysis system for use with diaries or food frequency questionnaires. Community Health Stud. 1984; 8: 11-8.

5 Rohan TE, Potter JD. Retrospective assessment of dietary intake. Am. J. Epidemiol. 1984; 120: 876-87.

6 Gliksman MD, Lazarus R, Wilson R, Leeder SR, Koutts J. The Western Sydney stroke risk in the elderly study. A 5-year prospective study. Ann. Epidemiol. 1994; 4: 59-66.

7 Hodge L, Salome CM, Peat JK, Haby MM, Xuan W, Woolcock AJ. Consumption of oily fish and childhood asthma risk. Med. J. Aust. 1996; 164: 137-40.

8 Baghurst PA, McMichael AJ, Slavotinek AH, Baghurst K, Boyle P, Walker AM. A case-control study of diet and cancer of the pancreas. Am. J. Epidemiol. 1991; 134: 167-79.

9 Potter JD, McMichael AJ. Diet and cancer of the colon and rectum: a case-control study. J. Natl. Cancer Inst. 1986; 76: 557-69.

10 Steinmetz KA, Potter JD. Food group consumption and colon cancer in the Adelaide case-control study. I. Vegetables and fruit. Int. J. Cancer 1993; 53: 711-9.

11 Kune GA, Bannerman S, Watson LF. Attributable risk for diet, alcohol, and family history in the Melbourne Colorectal Cancer Study. Nutr. Cancer 1992; 18: 231-5.

12 Rohan TE, McMichael AJ, Baghurst KI. A population-based case-control study of diet and breast cancer in Australia. Am. J. Epidemiol. 1988; 128: 478-89. 
13 Baghurst KI, Record SJ. Intake and sources in selected Australian subpopulations, of dietary constituents implicated in the etiology of chronic diseases. J. Food Nutr. 1983; 40 $1-15$.

14 Rangan AM, Binns CW, Blight GD. Dietary iron and calcium intakes of female university students. Aust. J. Nutr. Diet. 1997; 54: 110-6.

15 Baghurst KI, Dreosti IE, Syrette JA, Record SJ, Baghurst PA, Buckley RA. Zinc and magnesium status of Australian adults. Nutr. Res. 1991; 11: 23-32.

16 Baghurst KI. Dietary intakes of a free living elderly population. Proc. Nutr. Soc. Aust. 1985; 10: 174.

17 Baghurst KI, Record SJ. The vitamin and mineral intake of a free-living young elderly Australian population in relation to total diet and supplementation practices. Hum. Nutr. Appl. Nutr. 1987; 41A: 327-37.

18 Horwath CC. Dietary survey of a large random sample of elderly people: energy and nutrient intakes. Nutr. Res. 1989; 9: 479-92

19 Syrette JA, Baghurst KI. Dairy products and nutrient intake in ten year old children. Proc. Nutr. Soc. Aust. 1988; 13: 145 .

20 Baghurst KI, Record SJ, Baghurst PA, Syrette JA, Crawford D, Worsley A. Sociodemographic determinants in Australia of the intake of food and nutrients implicated in cancer aetiology. Med.J. Aust. 1990; 153: 444-52.

21 Baghurst KI, Crawford DA, Worsley A, Record SJ. The Victorian Nutrition Survey - intakes and sources of dietary fats and cholesterol in the Victorian population. Med.J. Aust. 1988; 149: 12-20.

22 Horwath CC, Worsley A. Dietary habits of elderly persons with diabetes. J. Am. Diet. Assoc. 1991; 91: 553-7.

23 Riley MD, Blizzard L. Comparative validity of a food frequency questionnaire for adults with IDDM. Diabetes Care 1995; 18: 1249-54.

24 Barratt A, Reznik R, Irwig L, Cuff A, Simpson JM, Oldenburg $\mathrm{B}$, et al. Work-site cholesterol screening and dietary intervention: the staff healthy heart project. Am. J. Public Health 1994; 84: 779-82.

25 Horwarth CC. Dietary changes reported by a random sample of elderly people. J. Nutr. Elderly 1992; 12: 13-27.

26 Baghurst KI, Baghurst PA, Record SJ. Demographic and nutritional profiles of people consuming varying levels of added sugars. Nutr. Res. 1992; 12: 1455-65.

27 Rohan TE, Record SJ, Cook MG. Repeatability of estimates of nutrient and energy intake: the quantitative food frequency approach. Nutr. Res. 1987; 7: 125-37.

28 Tassie K, Baghurst K, Record S, Nordin B. Usual dietary intake of middle-aged women using the food frequency method. In: Wahlqvist M, Truswell A, eds. Recent Advances in Clinical Nutrition. London: John Libbey, 1985.
29 Gelissen IC, Roberts DCK. Comparison of estimated nutrient intake by two methods: validation of a food frequency questionnaire. J. Hum. Nutr. Diet. 1992; 5: 215-23.

30 Amanatidis S, Mackerras D, Simpson JM. Comparison of two frequency questionnaires for quantifying fruit and vegetable intake. Public Health Nutr. 2001; 4: 233-9.

31 Armstrong BK, White E, Saracci R. Principles of Exposure Measurement in Epidemiology. New York: Oxford University Press, 1992

32 Bland JM, Altman DG. Measuring agreement in method comparison studies. Stat. Meth. Med. Res. 1999; 8: 135-60.

33 Musk AW, de Klerk NH, Ambrosini GL, Eccles JL, Hansen J, Olsen NJ, et al. Vitamin A and cancer prevention I: observations in workers previously exposed to asbestos at Wittenoom, Western Australia. Int. J. Cancer 1998; 75 335-61.

34 Goodsell C. Diet/1 Nutrient Analysis Software, Edition 3.10. Brisbane: Xyris Software, 1991.

35 Lewis J, Holt R. NUTTAB91-92: Commonwealth Government of Australia. Canberra: National Food Authority, 1992.

36 Schofield WN. Predicting basal metabolic rate, new standards and review of previous work. Hum. Nutr. Clin. Nutr. 1985; 39(Suppl. 1): 5-41.

37 Kristal AR, Cohen JH, Qu P, Stanford JL. Associations of energy, fat, calcium, and vitamin D with prostate cancer risk. Cancer Epidemiol. Biomark. Prev. 2002; 11: 719-25.

38 Bland MJ, Altman DG. Statistical methods for assessing agreement between two methods of clinical assessment. Lancet 1986; 1(8476): 307-10.

39 Chinn S. The assessment of methods of measurement. Stat. Med. 1990; 9: 351-62.

40 SAS Institute, Inc.. The SAS System for Windows, Version 8.01. Cary, NC: SAS Institute Inc., 2000.

41 Ambrosini GL, de Klerk NH, Musk AW, Mackerras D. Agreement between a brief food frequency questionnaire and diet records using two statistical methods. Public Health Nutr. 2001; 4: 255-64.

42 Food and Agriculture Organization (FAO)/World Health Organization (WHO)/United Nations University (UNU). Energy and Protein Requirements. Report of a Joint Expert Consultation. WHO Technical Report Series No. 724. Geneva: FAO/WHO/UNU, 1985.

43 Wheeler CE, Rutishauser IHE, O'Dea K. Comparison of nutrient intake data from two food frequency questionnaires and weighed records. Aust. J. Nutr. Diet. 1995; 52: 140-8.

44 Hodge A, Patterson AJ, Brown WJ, Ireland P, Giles G. The Anti Cancer Council of Victoria FFQ: relative validity of nutrient intakes compared with weighed food records in young to middle-aged women in a study of iron supplementation. Aust. NZ J. Public Health 2000; 24: 576-83. 